\title{
NONEXPANSIVE RETRACTS IN BANACH SPACES
}

\author{
EVA KOPECKÁ \\ Institute of Mathematics, Czech Academy of Sciences \\ Žitná 25, 11567 Prague, Czech Republic \\ and \\ Institut für Analysis, Johannes Kepler Universität, A-4040 Linz, Austria \\ E-mail: eva@bayou.uni-linz.ac.at \\ SIMEON REICH \\ Department of Mathematics, The Technion-Israel Institute of Technology \\ 32000 Haifa, Israel \\ E-mail: sreich@tx.technion.ac.il
}

\begin{abstract}
We study various aspects of nonexpansive retracts and retractions in certain Banach and metric spaces, with special emphasis on the compact nonexpansive envelope property.

1. Introduction. Let $X$ and $Y$ be two (real) Banach spaces, $A$ a closed subset of $X$, and $f: A \rightarrow Y$ continuous. According to a special case of Dugundji's generalization of Tietze's classical extension theorem [D, p. 357] (see also [BP, p. 58]), the mapping $f$ has a continuous extension $\tilde{f}: X \rightarrow Y$ to all of $X$ such that $\tilde{f}(X)$ is contained in the convex hull of $f(A)$. It immediately follows that any closed convex subset $C$ of $X$ is a retract of $X$. That is, there is a continuous mapping $R: X \rightarrow C$ such that $R x=x$ for each $x \in C$. If $X$ happens to be a Hilbert space $H$, then the nearest point projection $P: H \rightarrow C$ shows that $C$ is, in fact, a nonexpansive retract of $X$. In other words, there exists a nonexpansive (that is, 1-Lipschitz) mapping $R: X \rightarrow C$ which coincides with the identity on $C$. However, it is known $[\mathrm{P}$, p. 795] that if nearest point projections are nonexpansive whenever they exist for closed convex subsets $C$ of a Banach space $X$ with dimension at least three, then $X$ must be a Hilbert space. Moreover, it is also known $[\mathrm{R} 2$, p. 381] that if every closed convex subset of a Banach space $X$ with $\operatorname{dim}(X) \geq 3$ is a nonexpansive retract of $X$, then $X$ is necessarily a Hilbert space.
\end{abstract}

2000 Mathematics Subject Classification: Primary 46B50, 47H09, 52A05; Secondary 46B20, $52 \mathrm{~A} 55$.

Key words and phrases: Banach space, nonexpansive retraction, optimal set, sunny nonexpansive retraction.

The paper is in final form and no version of it will be published elsewhere. 
Thus it is of interest to determine, given a Banach space, what its (convex) nonexpansive retracts are. Indeed, the (convex) nonexpansive retracts of two-dimensional spaces $[\mathrm{G}], \ell_{p}$ spaces, $1<p<\infty, p \neq 2[\mathrm{H}],[\mathrm{DE}], L^{1}(0,1)[\mathrm{E} 1], \ell_{1}[\mathrm{~W}], L^{p}(0,1)$, $1<p<\infty, p \neq 2[\mathrm{E} 2]$, and of certain Köthe sequence spaces [KL], have all been characterized. At this point we mention only the following two results.

Recall that a retraction $R: X \rightarrow C$ is said to be sunny (a projection in [B2]) if for each $x \in X, R x=v$ implies that

$$
R(v+r(x-v))=v \quad \text { for all } r \geq 0
$$

[R1, p. 64]. If there exists a retraction $R: X \rightarrow C$ which is both sunny and nonexpansive, then we say that $C$ is a sunny nonexpansive retract of $X$.

THEOREM $1.1([\mathrm{~K}])$. Every closed convex subset of a two-dimensional Banach space $X$ is a sunny nonexpansive retract of $X$.

THEOREM $1.2([\mathrm{~B} 3])$. In any non-Hilbert Banach space $X$ of dimension greater than or equal to three, no bounded, smooth, closed and convex subset of $X$ with a nonempty interior is a nonexpansive retract of $X$.

Nonexpansive retracts have been studied in several contexts; we mention, for instance, fixed point theory [B1], optimal sets [Be], extension problems [R2], and nonlinear semigroup theory [R3].

Another interesting motivation comes from the Plateau problem in the calculus of variations which is concerned with finding minimal surfaces with a given boundary. It follows from [AK, Theorem 10.6] and [We, Corollary 1.3] that if $X$ is a dual Banach space with a separable predual, then the generalized Plateau problem can be solved in $X$. That is, for every boundary $B$ with compact support, there is a minimal surface with this given boundary. One of the crucial ingredients in the proof is that of finding a minimizing sequence of "surfaces", called metric integral currents, with this boundary $B$, such that all of them remain inside a compact set. In $[\mathrm{AK}]$ this compact set is the closed unit ball of $X$ with the weak-star topology, which in the case of a separable predual is strong enough to ensure that limit currents are also nice (rectifiable). Another possibility is to "push", so to speak, the minimizing sequence of "surfaces" inside a norm compact set by means of a nonexpansive retraction if such a retraction is available; in $[\mathrm{AK}]$ the authors proceed in this way when the Banach space $X$ is a $C(K)$ space, where $K$ is any compact metric space.

We say that a Banach space $X$ has the compact nonexpansive envelope property (abbreviation: $\mathrm{CNEP}$ ) if every compact subset of $X$ is contained in a compact nonexpansive retract of $X$.

Since the Plateau problem has a solution in any Banach space with the CNEP, it is of interest to determine which Banach spaces have this property. We address this problem in the next section of this note, where we also examine the Hilbert ball with its hyperbolic metric. In this connection we observe that although Theorem 10.6 in [AK] has recently been extended to all dual Banach spaces [We, Theorem 1.5], the Plateau problem is still unsolved for general Banach spaces. We also remark in passing that certain nonexpansive retractions are used to solve the Plateau problem in $L^{1}\left(\mathbb{R}^{m}\right)[\mathrm{AK}]$, 
dual Banach spaces [We] and Hadamard spaces [We]. In Section 3 we show that $X=\ell_{1}$ lacks the CNEP and that its closed unit ball is not a nonexpansive retract of $X$. In Section 4 we briefly discuss the relations between the optimal sets introduced in $[\mathrm{BM}]$ and nonexpansive retracts. The last section of our paper is devoted to sunny nonexpansive retractions.

In the sequel we will sometimes use the abbreviation NR to refer to both nonexpansive retractions and nonexpansive retracts.

2. Envelopes. In this section we study the CNEP in various Banach spaces. We also consider the Hilbert ball and its powers.

Recall that a norm $\|\cdot\|$ on $\mathbb{R}^{d}$ is said to be absolute if

$$
\left\|\left(x_{1}, x_{2}, \ldots, x_{d}\right)\right\|=\left\|\left(\left|x_{1}\right|,\left|x_{2}\right|, \ldots,\left|x_{d}\right|\right)\right\|
$$

for all $\left(x_{1}, x_{2}, \ldots, x_{d}\right) \in \mathbb{R}^{d}$. It is called monotone if whenever $0 \leq x \leq y$ coordinatewise, it follows that $\|x\| \leq\|y\|$.

Proposition 2.1. Let the Banach space $X$ be $\mathbb{R}^{d}$ with a norm $\|\cdot\|$ that is both absolute and monotone. Then $X$ has the CNEP.

Proof. For a positive number $a$, let $q_{a}: \mathbb{R} \rightarrow \mathbb{R}$ be defined by $q_{a}(x)=x$ if $|x| \leq a$ and by $q_{a}(x)=(\operatorname{sgn} x) a$ if $|x| \geq a$. Then $q_{a}$ is nonexpansive. Now let $a_{1}, a_{2}, \ldots, a_{d}$ be $d$ positive numbers and consider the parallelepiped

$$
C:=\left\{\left(x_{1}, x_{2}, \ldots, x_{d}\right) \in \mathbb{R}^{d}:\left|x_{j}\right| \leq a_{j}, 1 \leq j \leq d\right\} .
$$

It is not difficult to see that the mapping $Q: X \rightarrow C$ defined by

$$
Q\left(x_{1}, x_{2}, \ldots, x_{d}\right)=\left(q_{a_{1}}\left(x_{1}\right), q_{a_{2}}\left(x_{2}\right), \ldots, q_{a_{d}}\left(x_{d}\right)\right)
$$

is a nonexpansive retraction. Since any compact subset of $X$ is obviously contained in such a compact parallelepiped, we see that $X$ does indeed have the CNEP.

As usual, we denote by $c_{0}$ the Banach space of all real sequences tending to zero, endowed with the supremum norm.

Proposition 2.2. The space $c_{0}$ has the CNEP.

Proof. Let $K$ be a compact subset of $c_{0}$. Then it is not difficult to see that there is a sequence of positive numbers $\left(a_{1}, a_{2}, \ldots, a_{n}, \ldots\right) \in c_{0}$ such that if $\left(x_{1}, x_{2}, \ldots, x_{n}, \ldots\right) \in K$, then $\left|x_{j}\right| \leq a_{j}$ for all $j=1,2, \ldots$.

The set $C \subset c_{0}$, defined by

$$
C:=\left\{\left(x_{1}, x_{2}, \ldots, x_{n}, \ldots\right) \in c_{0}:\left|x_{j}\right| \leq a_{j}, j=1,2, \ldots\right\},
$$

contains $K$ and is also compact. Since the mapping $Q: c_{0} \rightarrow C$ defined by

$$
Q\left(x_{1}, x_{2}, \ldots, x_{n}, \ldots\right)=\left(q_{a_{1}}\left(x_{1}\right), q_{a_{2}}\left(x_{2}\right), \ldots, q_{a_{n}}\left(x_{n}\right), \ldots\right)
$$

is a nonexpansive retraction onto $C$, the result follows.

Essentially the same proof provides us with more examples of spaces with the CNEP. If $(E,|\cdot|)$ is a Banach space, we denote by $c_{0}(E)$ the space of all $E$-valued null sequences, endowed with the norm $\left\|\left(x_{1}, x_{2}, \ldots, x_{n}, \ldots\right)\right\|:=\sup \left\{\left|x_{n}\right|: n=1,2, \ldots\right\}$. 
Proposition 2.3. Let $E$ be a finite-dimensional Banach space. If the closed unit ball of $E$ is a nonexpansive retract of $E$, then $c_{0}(E)$ has the CNEP.

Proof. In the proof of Proposition 2.2 we replace the mapping $q_{a}: \mathbb{R} \rightarrow[-a, a]$ with the mapping $\tilde{q}_{a}: E \rightarrow B(0, a)$, defined by $\tilde{q}_{a}(x):=a \tilde{q}(x / a)$, where $\tilde{q}: E \rightarrow B(0,1)$ is an NR of $E$ onto the closed unit ball $B(0,1)$ of $E$, and $B(0, a)$ is the closed ball of radius $a$ centered at the origin.

This proposition applies, for example, to two-dimensional spaces (Theorem 1.1), $\ell_{2}^{d}$ and $\ell_{\infty}^{d}$. A similar proof shows that the finite power $E^{d}$, when equipped with a norm that is both absolute and monotone, also has the CNEP. This is also true for any finite Cartesian product of spaces with the CNEP. We also note that since the closed convex hull of a compact subset of a Banach space is also compact, every Hilbert space clearly has the CNEP.

On the other hand, we are now going to show that no $\ell_{p}$ space, $1<p<\infty, p \neq 2$, has the CNEP $[\mathrm{Ko}]$.

To this end, we first recall a characterization of the NR's in $\ell_{p}^{d}, d \geq 3[\mathrm{H}]$, and $\ell_{p}$, $1<p<\infty, p \neq 2$ [DE, E2].

TheOREm 2.4. Let $X$ be either $\ell_{p}^{d}, d \geq 3$, or $\ell_{p}, 1<p<\infty, p \neq 2$. A proper subset of $X$ is an NR of $X$ if and only if it is the intersection of a family of closed half-spaces of the form

$$
\left\{x \in X: a x_{i}+b x_{j} \leq c\right\}, \quad i \neq j .
$$

For a subset $D$ of a Banach space $(X,\|\cdot\|)$, we denote $\sup \{\|x\|: x \in D\}$ by $\operatorname{rad}(D)$. Lemma 2.5. Let $X$ be the $d$-dimensional Banach space $\ell_{p}^{d}$, where $d \geq 3$ and $1<p<\infty$, $p \neq 2$, and let $B(0, r) \subset X$ be the closed ball of radius $r>0$ centered at the origin. If $A \subset X$ is an $N R$ that contains $B(0, r)$, then $\operatorname{rad}(A) \geq(r / 2) d^{1 / p}$.

Proof. Consider the points $z_{t}=t(1,1, \ldots) \in X$, where $t \in \mathbb{R}$. If such a point does not belong to $A$, then by Theorem 2.4, neither does it belong to a closed half-space of the form $\left\{x \in X: a x_{i}+b x_{j} \leq c\right\}, i \neq j$, which, however, does contain $B(0, r)$. Therefore $a x_{i}+b x_{j} \leq c<t(a+b)$ for all $x_{i}$ and $x_{j}$ such that $\left|x_{i}\right|^{p}+\left|x_{j}\right|^{p} \leq r^{p}$. Choosing $x_{i}=x_{j}=r \operatorname{sgn}(a+b) 2^{-1 / p}$, we obtain $r|a+b|<t(a+b) 2^{1 / p}<2 t(a+b)$. Hence $|t|>r / 2$. Thus all the points $z_{t}$ with $|t| \leq r / 2$ belong to $A$ and the result follows.

We now recall a consequence of Theorem 2 in [B1].

THEOREM 2.6. Let $C$ be a closed convex subset of a reflexive Banach space every bounded, closed and convex subset of which has the fixed point property for nonexpansive mappings. If $T: C \rightarrow C$ is nonexpansive, then its fixed point set is an NR of $C$.

We denote the set of natural numbers by $\mathbb{N}$.

TheOREM 2.7. The spaces $\ell_{p}, 1<p<\infty, p \neq 2$, do not have the CNEP.

Proof. Choose a positive sequence $\left\{r_{n}: n \in \mathbb{N}\right\}$ and a sequence $\left\{d_{n}: n \in \mathbb{N}\right\} \subset \mathbb{N}, d_{n} \geq 3$, so that $\sum_{n=1}^{\infty} r_{n}^{p}<\infty$, but $r_{n} d_{n}^{1 / p} \rightarrow \infty$ as $n \rightarrow \infty$. Let $\left\{e^{n}: n \in \mathbb{N}\right\}$ be the standard basis in $\ell_{p}$. For each $n \in \mathbb{N}$, let $P_{n}: \ell_{p} \rightarrow \ell_{p}^{d_{n}}$ be the linear operator of norm one which assigns 
to each $x=\left(x_{1}, x_{2}, \ldots\right) \in \ell_{p}$ the point $\left(x_{s_{n-1}+1}, x_{s_{n-1}+2}, \ldots, x_{s_{n-1}+d_{n}}\right) \in \ell_{p}^{d_{n}}$, where $s_{n}:=\sum_{j=1}^{n} d_{j}$. Consider the set

$$
K:=\left\{x \in \ell_{p}: P_{n} x \in B_{n}\left(0, r_{n}\right) \text { for all } n \in \mathbb{N}\right\},
$$

where $B_{n}\left(0, r_{n}\right) \subset \ell_{p}^{d_{n}}$ is the closed ball of radius $r_{n}$ centered at the origin of $\ell_{p}^{d_{n}}$. This set is compact because $\sum_{n=1}^{\infty} r_{n}^{p}<\infty$. Suppose that $K \subset D \subset \ell_{p}$ is an NR of $\ell_{p}$ and let $R: \ell_{p} \rightarrow D$ be a nonexpansive retraction. Let the nonexpansive mapping $T_{n}: \ell_{p}^{d_{n}} \rightarrow \ell_{p}^{d_{n}}$ be defined as follows: if $x \in \ell_{p}^{d_{n}}$, then

$$
T_{n} x:=P_{n} R\left(\sum_{j=1}^{d_{n}} x_{j} e^{s_{n-1}+j}\right) \in \ell_{p}^{d_{n}} .
$$

If $y \in B_{n}\left(0, r_{n}\right)$, then $\sum_{j=1}^{d_{n}} y_{j} e^{s_{n-1}+j}$ belongs to $K \subset D$ and therefore $T_{n} y=y$. Thus the fixed point set $F\left(T_{n}\right)$ of $T_{n}$ contains $B_{n}\left(0, r_{n}\right)$. Since it is an NR of $\ell_{p}^{d_{n}}$ by Theorem 2.6, it follows from Lemma 2.5 that $\operatorname{rad}\left(F\left(T_{n}\right)\right) \geq\left(r_{n} / 2\right) d_{n}^{1 / p}$. Since $F\left(T_{n}\right) \subset P_{n}(D)$, we see that

$$
\operatorname{rad}(D) \geq \operatorname{rad}\left(P_{n}(D)\right) \geq\left(r_{n} / 2\right) d_{n}^{1 / p} .
$$

Thus $D$ cannot be even bounded.

This proof of Theorem 2.7 is somewhat different from the original one [Ko], where no use is made of Theorem 2.6; instead, the characterization of Theorem 2.4 is applied directly to $\ell_{p}$ and not just to $\ell_{p}^{d}$.

We will soon present more examples of spaces without the CNEP. However, at this point we show how the argument employed in the proof of Theorem 2.7 can also be used to construct new such examples out of old ones.

THEOREM 2.8. Let $E$ be a reflexive Banach space every bounded, closed and convex subset of which has the fixed point property for nonexpansive mappings. If $E$ does not have the $C N E P$, then neither does $c_{0}(E)$.

Proof. Let $B \subset E$ be a compact subset of $E$ which is not contained in any compact NR of $E$. Denote $c_{0}(E)$ by $X$, and for each $n \in \mathbb{N}$, let $P_{n}: X \rightarrow E$ be the linear operator of norm one which assigns to each $x \in c_{0}(E)$ its $n$-th coordinate. Consider the compact subset $K$ of $X$ defined by $K:=\left\{x \in X: P_{1} x \in B, P_{n} x=0, n \geq 2\right\}$ and suppose $K \subset D \subset X$ is an NR. Let $R: X \rightarrow D$ be a nonexpansive retraction and define a mapping $T: E \rightarrow E$ as follows: given $x \in E$, let $T x:=P_{1} R(x, 0, \ldots, 0, \ldots)$. If $x \in B$, then $(x, 0, \ldots, 0, \ldots) \in K \subset D$ and $T x=x$. Thus the fixed point set $F(T)$ of $T$ contains $B$. Since $T$ is nonexpansive, $F(T)$ is an NR of $E$ by Theorem 2.6. Hence it is not compact. Since $F(T) \subset P_{1}(D)$, neither are $P_{1}(D)$ and $D$ itself.

Note that the same argument shows that in Theorem 2.8, $c_{0}(E)$ may be replaced with $\ell_{p}(E)$.

It follows from the Hahn-Banach theorem that any one-dimensional subspace of any Banach space is the range of a norm one linear projection. There exist, however, finite- 
dimensional, strictly convex spaces, of dimension greater than or equal to three, in which the only norm one linear projections are the identity and the projections onto lines [Bo].

We now observe that all such spaces lack the CNEP. To see this, let $B$ denote the closed unit ball of such a space $E$, and suppose $C$ is a compact NR of $E$ which contains $B$. Then $C$ is bounded, closed, convex and has a nonempty interior. Since its Minkowski functional is Gâteaux differentiable on a dense subset of $E$, it follows that the boundary of $C$ contains points of smoothness. Let $y_{0}$ be such a point and let $H+y_{0}$ be the unique supporting hyperplane at $y_{0}$. By assumption, the subspace $H$ is not the range of a linear projection of norm one. Therefore $C$ is not, after all, an NR of $E$ by Theorem 2 of [FK], which asserts that if the Banach space $E$ is reflexive and a convex NR of $E$ with a nonempty interior has at one of its boundary points $y_{0}$ a unique supporting hyperplane $H+y_{0}$, then the subspace $H$ is the range of a linear projection of norm one.

Instead of invoking Theorem 2 of $[\mathrm{FK}]$, one could also argue as follows: if $C$ were an NR of $E$, then the supporting hyperplane $H+y_{0}$ would be the common boundary of two closed half-spaces which would also be NR's of $E$ by [B1, Lemma 5]. It would then follow from [B1, Theorem 5] that the subspace $H$ is an NR too, and hence, in fact, the range of a linear projection of norm one, a contradiction.

Another class of examples of spaces without the CNEP can be obtained, as pointed out by S. Wenger, by recalling the following special case of a theorem of L. Veselý [V].

THEOREM 2.9. If $X$ is a nonreflexive Banach space, then it has an equivalent norm $|\cdot|$ and contains a three point set $K=\left\{y_{1}, y_{2}, y_{3}\right\}$ such that the functional $f:(X,|\cdot|) \rightarrow \mathbb{R}^{+}$ defined by

$$
f(x):=\left|x-y_{1}\right|+\left|x-y_{2}\right|+\left|x-y_{3}\right|
$$

does not attain its infimum on $(X,|\cdot|)$.

Since it is clear that the compact set $K \subset X$ cannot be contained in a compact NR of $(X,|\cdot|)$, it follows that $(X,|\cdot|)$ lacks the CNEP.

Returning to reflexive spaces, we recall [B1, Theorem 5] that if $X$ is reflexive and strictly convex, then the class of nonexpansive retracts is closed under arbitrary intersections. Therefore we can define, for each nonempty subset $D$ of $X$, its nonexpansive hull $N(D)$ as the intersection of all the NR's containing it. Thus we see that a reflexive and strictly convex Banach space $X$ has the CNEP if and only if $N(K)$ is compact for each compact subset of $X$.

The definition of the CNEP can be extended to all metric spaces in an obvious way. To present examples of nonlinear metric spaces with the CNEP, we first consider the Hilbert ball $\mathbb{B}$ equipped with the hyperbolic metric $\rho: \mathbb{B} \times \mathbb{B} \rightarrow \mathbb{R}^{+}[\mathrm{GR}$, pp. 108-110]. Given a subset $D$ of $\mathbb{B}$, we define its $\rho$-closed convex hull, $\rho$-clco $(D)$, to be the intersection of all the $\rho$-closed and $\rho$-convex subsets of $\mathbb{B}$ which contain $D$.

Since each $\rho$-ball is $\rho$-convex, it is clear that if $D$ is $\rho$-bounded, so is $\rho$-clco $(D)$. Therefore it is obvious that if $K \subset \mathbb{B}$ is compact and $\mathbb{B}$ is finite-dimensional, then $\rho$-clco $(K)$ is also compact. Since $\rho$-convexity in the real Hilbert ball $(\widetilde{\mathbb{B}}, \rho)[\mathrm{GR}$, p. 148$]$ coincides with linear convexity, this is also true for compact subsets of $(\widetilde{\mathbb{B}}, \rho)$. Finally, since every $\rho$-closed and $\rho$-convex subset of $(\mathbb{B}, \rho)$ is a $\rho$-Chebyshev set, and the nearest point projection of $\mathbb{B}$ 
onto it is $\rho$-nonexpansive [GR, pp. 108-110], we conclude that each finite-dimensional Hilbert ball, as well as any real Hilbert ball, all have the CNEP. As a matter of fact, this turns out to be true for any Hilbert ball whatsoever.

TheOREM 2.10. Every Hilbert ball $(\mathbb{B}, \rho)$ has the CNEP.

Proof. For each subset $D$ of $\mathbb{B}$, we define $C_{1}(D)$ to be the union of all the metric segments $[x, y] \subset \mathbb{B}$ with endpoints $x$ and $y$ in $D$. It is clear that $D$ is $\rho$-convex if and only if $D=C_{1}(D)$. It is also not difficult to see that $\rho$-clco $(D)$ is the closure of the union $\bigcup\left\{C_{n}(D): n=0,1,2, \ldots\right\}$, where $C_{0}(D):=D$ and $C_{n+1}(D):=C_{1}\left(C_{n}(D)\right), n \geq 1$.

Now suppose $D=K$ is compact. To show that $\rho$-clco $(K)$ is also compact, we need to show that it is totally bounded. This means that for each $\varepsilon>0$, we have to find a finite $\varepsilon$-net for $\rho$-clco $(K)$. To this end, we first find a finite $(\varepsilon / 2)$-net $D=\left\{k_{1}, k_{2}, \ldots, k_{N}\right\} \subset K$ and consider $\rho$-clco $(D)$. This last set is compact because it is contained in a finitedimensional Hilbert ball. Next we compare $\bigcup\left\{C_{n}(K): n=0,1,2, \ldots\right\}$ with $\bigcup\left\{C_{n}(D)\right.$ : $n=0,1,2, \ldots\}$. We claim that for each $n=0,1,2, \ldots$, each point in $C_{n}(K)$ is within $\varepsilon / 2$ of a point in $C_{n}(D)$. Indeed, this is obviously true for $n=0$ by the definition of $D$. If this is true for $C_{n}(K)$ and $C_{n}(D)$, then the inequality

$$
\rho((1-t) x \oplus t y,(1-t) z \oplus t w) \leq(1-t) \rho(x, z)+t \rho(y, w),
$$

where $0 \leq t \leq 1$ and $x, y, z, w$ are all in $\mathbb{B}$ [GR, p. 104], implies that it is also true for $C_{n+1}(K)=C_{1}\left(C_{n}(K)\right)$ and $C_{n+1}(D)=C_{1}\left(C_{n}(D)\right)$. So each point in $\rho$-clco $(K)$ is within $\varepsilon / 2$ of a point in $\rho$-clco $(D)$. Thus any $(\varepsilon / 2)$-net in $\rho$-clco $(D)$ is an $\varepsilon$-net for $\rho$-clco $(K)$.

This proof of Theorem 2.10 works equally well in any Hadamard space in which the $\rho$-closed convex hull of a finite number of points is compact. It follows then that the Plateau problem can be solved in such spaces. Unfortunately, it is not known which Hadamard spaces have this property. However, it is shown in [We, Theorem 1.6] that Plateau's problem can be solved in every Hadamard space (regardless of whether it has this property or not).

For each $d \in \mathbb{N}$, let $\left(H^{d},|\cdot|_{\infty}\right)$ denote the $d$-th power of a complex Hilbert space $H$, endowed with the maximum norm, and let $\left(X, \rho_{\infty}\right)$ be the open unit ball of $H^{d}$, equipped with its Kobayashi metric. Then $X=\mathbb{B}^{d}$, and

$$
\rho_{\infty}(x, y)=\max \left\{\rho\left(x_{j}, y_{j}\right): 1 \leq j \leq d\right\},
$$

where $\mathbb{B}$ is the Hilbert ball and $\rho$ is its hyperbolic metric. We also note that the discussion on p. 91 of $[\mathrm{GR}]$ implies that if $D$ is a bounded domain in a complex Banach space $(E,|\cdot|)$ and $\rho$ is any metric assigned to it by a Schwarz-Pick system of pseudometrics, then a subset $K$ of $(D, \rho)$ is compact if and only if it is a compact subset of $(E,|\cdot|)$.

Proposition 2.11. The metric space $\left(\mathbb{B}^{d}, \rho_{\infty}\right)$ has the CNEP for each $d \in \mathbb{N}$.

Proof. Let $K \subset \mathbb{B}^{d}$ be a compact subset of $\mathbb{B}^{d}$. For each $1 \leq j \leq d$, let $P_{j}: H^{d} \rightarrow H$ assign to each $x \in H^{d}$ its $j$-th coordinate, set $C_{j}:=\rho$-clco $\left(P_{j}(K)\right)$, and let $R_{j}: \mathbb{B} \rightarrow C_{j}$ be the nearest point projection of $\mathbb{B}$ onto $C_{j}$. Then each $C_{j}$ is compact by the proof of Theorem 2.10 and the Cartesian product $C=C_{1} \times C_{2} \times \ldots \times C_{d}$ is a compact subset of $\mathbb{B}^{d}$ which contains $K$. Since the mapping $R: \mathbb{B}^{d} \rightarrow C$ defined by $R\left(x_{1}, x_{2}, \ldots, x_{d}\right):=$ $\left(R_{1} x_{1}, R_{2} x_{2}, \ldots, R_{d} x_{d}\right)$ is a $\rho$-nonexpansive retraction of $\mathbb{B}^{d}$ onto $C$, the result follows. 
Let $\mathbb{B}_{H}^{\infty}$ be the open unit ball of $\ell_{\infty}(H)$. It is known that the Kobayashi metric $\rho_{\infty}$ on $\mathbb{B}_{H}^{\infty} \times \mathbb{B}_{H}^{\infty}$ is given by

$$
\rho_{\infty}(x, y)=\sup \left\{\rho\left(x_{j}, y_{j}\right): j \in \mathbb{N}\right\},
$$

where $\rho: \mathbb{B} \times \mathbb{B} \rightarrow \mathbb{R}^{+}$is again the hyperbolic metric on $\mathbb{B} \times \mathbb{B}$. Similar arguments to those used in the proofs of Proposition 2.3 and Proposition 2.11 show that the metric space $\left(\mathbb{B}_{H}^{\infty} \cap c_{0}(H), \rho_{\infty}\right)$ also has the CNEP.

3. Balls. Consider the closed unit balls $B_{p}(0,1)=B_{p}$ of the $\ell_{p}$ spaces, $1 \leq p \leq \infty$. It follows from Theorem 1.2 that no $B_{p}, 1<p<\infty, p \neq 2$, is an NR of $\ell_{p}$. On the other hand, the arguments of the proofs of Propositions 2.1 and 2.2 show that $B_{\infty}$ is an NR of $\ell_{\infty}$. Thus it is natural to ask (as did Y. Gordon) if $B_{1}$ is an NR of $\ell_{1}$. In this section we answer this question in the negative.

We begin with Westphal's characterization of the nonexpansive retracts of $\ell_{1}[\mathrm{~W}]$. Recall that a nonempty subset $C$ of $\ell_{1}$ is called a cylinder set with base $B$ in the plane $\left[e^{i}, e^{j}\right]$ spanned by the unit vectors $e^{i}$ and $e^{j}, i<j$, if there is a subset $B$ of $\left[e^{i}, e^{j}\right]$ such that $C$ is the direct sum of $B$ and $D_{i j}=\left\{x \in \ell_{1}: x_{i}=x_{j}=0\right\}$. A subset $E$ of a Banach space $(X,|\cdot|)$ is said to be metrically convex if for each pair of distinct points $x_{1}, x_{2}$ of $E$, there exists a point $x_{3}$ of $E$, distinct from $x_{1}$ and $x_{2}$, such that $\left|x_{1}-x_{2}\right|=\left|x_{1}-x_{3}\right|+\left|x_{3}-x_{2}\right|$.

Theorem 3.1 ([W]). Let $X$ be either $\ell_{1}^{d}, d \geq 3$, or $\ell_{1}$. A proper subset of $X$ is an $N R$ of $X$ if and only if it is metrically convex and the intersection of a family of closed cylinder sets.

TheOREm 3.2. Let $X$ be either $\ell_{1}^{d}, d \geq 3$, or $\ell_{1}$. Then the closed unit ball of $X$ is not an NR of $X$.

Proof. Suppose the closed unit ball $B_{1}$ of $X$ is contained in an NR $A$ of $X$. If the point $z \in X$ is not in $A$, then by Theorem 3.1, there is a cylinder set $C$, which contains $B_{1}$, to which $z$ does not belong either. Let $C$ be the direct sum of a base $B \subset\left[e^{i}, e^{j}\right], i<j$, and $D_{i j}$. Since $C$ contains $B_{1}$, it follows that $\left|z_{i}\right|+\left|z_{j}\right|>1$. Thus $A$ contains all those points $x \in X$ for which $\left|x_{m}\right|+\left|x_{n}\right| \leq 1$ for all $m, n \in \mathbb{N}$. Choosing $x=\frac{1}{2}(1,1, \ldots)$, we see that $\operatorname{rad}(A) \geq \frac{1}{2} d \geq \frac{3}{2}>1$. Hence $A \neq B_{1}$, as claimed.

The proof of Theorem 3.2 also yields the following analog of Lemma 2.5.

Lemma 3.3. Let $X$ be the $d$-dimensional Banach space $\ell_{1}^{d}, d \geq 3$, and let $B(0, r) \subset X$ be the closed ball of radius $r>0$ centered at the origin. If $A \subset X$ is an NR that contains $B(0, r)$, then $\operatorname{rad}(A) \geq(r / 2) d$.

This last lemma, when combined with the construction and arguments used in the proof of Theorem 2.7, yields, in its turn, the following analog of that theorem.

THEOREM 3.4. The space $\ell_{1}$ lacks the CNEP.

As a matter of fact, the arguments used in the proofs of Lemma 2.5 and Theorem 3.2 can also be employed to show that no closed unit ball $B_{p}$ of $\ell_{p}, 1<p<\infty, p \neq 2$, is an NR of $\ell_{p}$. Indeed, if $B_{p}$ is contained in an NR $A$ of $\ell_{p}$, then these arguments show 
that all the points of the form $t\left(\sum_{j=1}^{d} e^{j}\right)$, where $|t| \leq \frac{1}{2}$ and $d \in \mathbb{N}$, belong to $A$. Thus $\operatorname{rad}(A) \geq(1 / 2) d^{1 / p}>1$ when $d>2^{p}$, and so $A \neq B_{p}$, as asserted.

To show that the closed unit balls of $\ell_{p}^{d}$, where $1<p<\infty, p \neq 2$, and $d \geq 3$, are not NR's, one can also make use of the following fact [FK, Proposition 1] (see also [R6, Proposition 3.4]): if $E$ is a non-Hilbert, strictly convex Banach space of (finite or infinite) dimension $d \geq 3$, then there exist subspaces of all finite dimensions $2 \leq k \leq d-1$, which are not the ranges of linear projections of norm one.

4. Optimal sets. In this section we briefly discuss the connections between the optimal sets introduced by Beauzamy and Maurey in [BM] and nonexpansive retracts.

Let $D$ be a nonempty subset of the Banach space $X$. A point $x \in X$ is said to be minimal with respect to $D$ if the condition

$$
|y-z| \leq|x-z| \text { for all } z \in D
$$

implies that $y=x$. We denote by $\operatorname{Min}(D)$ the set of all those points in $X$ which are minimal with respect to $D$. It is clear that $D \subset \operatorname{Min}(D)$ and that if $D_{1} \subset D_{2}$, then $\operatorname{Min}\left(D_{1}\right) \subset \operatorname{Min}\left(D_{2}\right)$. The set $D$ is said to be optimal if $\operatorname{Min}(D)=D$. Given an arbitrary nonempty subset $D$ of $X$, we define $M_{1}(D)$ to be $\operatorname{Min}(D)$ and then set $M_{n+1}(D):=$ $M_{1}\left(M_{n}(D)\right), n \geq 1$. The saturate $S(D)$ of $D[\mathrm{BM}]$ is defined to be the closure of the union $\bigcup\left\{M_{n}(D): n=1,2, \ldots\right\}$. We also define $O(D)$, the optimal hull of $D$, to be the intersection of all the closed optimal sets containing $D$. Note that $O(D)$ itself is both optimal [BM, Proposition III.2] and closed.

Proposition 4.1. If the Banach space $X$ is reflexive and strictly convex, then $S(D)=O(D)$ for all nonempty $D \subset X$.

Proof. Let $D$ be a nonempty subset of $X$. Since $S(D)$ is both optimal by Proposition III.4 on p. 121 of $[\mathrm{BM}]$ and closed by definition, it is clear that $O(D) \subset S(D)$. Conversely, we first note that obviously $D \subset O(D)$. Therefore $M_{1}(D)=\operatorname{Min}(D) \subset \operatorname{Min}(O(D))=O(D)$. If $M_{n}(D) \subset O(D)$ for some $n \geq 1$, then we also have $M_{n+1}(D)=M_{1}\left(M_{n}(D)\right) \subset$ $M_{1}(O(D))=O(D)$. Hence $\bigcup\left\{M_{n}(D): n=1,2, \ldots\right\}$ and $S(D)$ are contained in $O(D)$. Thus $S(D)=O(D)$, as claimed.

It is clear that the fixed point set of any nonexpansive self-mapping of $X$ and, in particular, any nonexpansive retract of $X$ are optimal. Here is a result in the converse direction.

THEOREM 4.2 ([Be]). If the Banach space $X$ is separable, reflexive, strictly convex and smooth, and $C \subset X$ is an optimal set with a nonempty interior, then $C$ is a nonexpansive retract of $X$.

Corollary 4.3. If the Banach space $X$ is separable, reflexive, strictly convex and smooth, and $D \subset X$ has a nonempty interior, then the nonexpansive hull $N(D)$ of $D$ coincides with $O(D)$, its optimal hull. 
This corollary yields another proof of Lemma 2.5. Indeed, it follows from the results of $[\mathrm{BM}]$ that in the setting of that lemma,

$$
S(B(0, r))=r\left\{x \in \mathbb{R}^{d}: \sup \left\{\left|x_{i}\right|^{p}+\left|x_{j}\right|^{p}: i \neq j\right\} \leq 1\right\} .
$$

Since $S(B(0, r))=N(B(0, r))$ by Corollary 4.3 and Proposition 4.1 , we see that if $A \subset X$ is an NR which contains $B(0, r)$, then $\left(\frac{1}{2} r\right)(1,1, \ldots, 1) \in A$. Hence $\operatorname{rad}(A) \geq\left(\frac{r}{2}\right) d^{1 / p}$, as asserted.

Corollary 4.3 is also obviously true whenever the Banach space $X$ has the following property: a subset of $X$ is optimal if and only if it is an NR. This is indeed known to be the case for the $\ell_{p}([\mathrm{DE}, \mathrm{E} 2])$ and $L^{p}[0,1]$ ([E2]) spaces, $1<p<\infty$, as well as for strictly convex, smooth and reflexive Köthe sequence spaces ([KL, Theorem 3.8]).

5. Sunny nonexpansive retracts. It is obvious that if $C$ is a nonexpansive retract of the Banach space $X$ and $T: C \rightarrow M$ is a Lipschitz mapping of $C$ into a metric space $M$, then $T$ can be extended to all of $X$ without increasing its Lipschitz constant. If $C$ happens to be a sunny nonexpansive retract of $X$, then the extension is much easier because it is constant along certain rays emanating from $C$. Other applications of sunny nonexpansive retracts (for instance, to extension problems for accretive operators, iterative methods for solving variational inequalities, and the convex feasibility problem) can be found, for example, in $[\mathrm{R} 2, \mathrm{R} 3, \mathrm{MR}]$ and the references therein. In this section we show that in many cases, nonexpansive retracts are, in fact, sunny nonexpansive retracts. We will sometimes use the abbreviation SNR to refer to both sunny nonexpansive retractions and sunny nonexpansive retracts.

We first observe that if a smooth Banach space $X$ has the CNEP, then every compact subset of $X$ is contained in a compact SNR of $X$.

Proposition 5.1. If $C$ is a compact $N R$ of a smooth Banach space $X$, then $C$ is, in fact, an SNR of $X$.

Proof. Let $R: X \rightarrow C$ be an NR and let $x$ be any point in $X$. Banach's fixed point theorem provides us, for each $0 \leq t<1$, a unique point $x_{t} \in X$ such that $x_{t}=(1-t) x+t R x_{t}$. Let $\left\{t_{n}\right\} \subset[0,1)$ be a sequence which converges to 1 , and denote the corresponding point $x_{t_{n}}$ by $x_{n}$. Since $C$ is compact, we may assume that the sequence $\left\{x_{n}\right\}$ converges strongly to $z \in C$. Let $J: X \rightarrow X^{*}$ be the normalized duality mapping of $X$. Since

$$
\left(1-t_{n}\right)\left(x-R x_{n}, J\left(x_{n}-y\right)\right)=\left(x_{n}-R x_{n}, J\left(x_{n}-y\right)\right) \geq 0
$$

for all $y \in C$ and $n \in \mathbb{N}$, we see that $(x-z, J(y-z)) \leq 0$ for all $y \in C$. Denoting $z$ by $Q x$ and invoking either [R1, p. 64] or [B2, p. 348], we now conclude that $Q: X \rightarrow C$ is the unique SNR of $X$ onto $C$.

Our next result was deduced in [R3] from a general theorem regarding the equivalence of resolvent consistency and convergence for nonlinear contractive algorithms. Here we present a somewhat simpler proof, which is also a variant of the so-called optimization method [R7].

Proposition 5.2. If $C$ is an NR of a reflexive Banach space $X$ with a uniformly Gâteaux differentiable norm, then it is, in fact, an SNR of X. 
Proof. Let $R: X \rightarrow C$ be an NR, $x$ a point in $X$, and $\left\{t_{n}\right\} \subset[0,1)$ a sequence which converges to 1 . For each $n \in \mathbb{N}$, denote by $x_{n} \in X$ the unique solution to the equation $w=\left(1-t_{n}\right) x+t_{n} R w$.

Fix a point $y$ in $C$. Since $\left|x_{n}-y\right| \leq|x-y|$ for all $n \in \mathbb{N}$, the sequence $\left\{x_{n}\right\}$ is bounded. Let LIM be a Banach limit and define $f: X \rightarrow[0, \infty)$ by

$$
f(z):=\operatorname{LIM}\left(\left\{\left|x_{n}-z\right|^{2}\right\}\right), \quad z \in X .
$$

Since the functional $f$ is continuous and convex, $f(z) \rightarrow \infty$ as $|z| \rightarrow \infty$, and $X$ is reflexive, $f$ attains its minimum over $X$, say at $u \in X$. Since $x_{n}-R x_{n} \rightarrow 0$ as $n \rightarrow \infty$, we have

$$
f(R z)=\operatorname{LIM}\left(\left\{\left|x_{n}-R z\right|^{2}\right\}\right)=\operatorname{LIM}\left(\left\{\left|R x_{n}-R z\right|^{2}\right\}\right) \leq \operatorname{LIM}\left(\left\{\left|x_{n}-z\right|^{2}\right\}\right)=f(z)
$$

for all $z \in X$, and therefore we may as well assume that $u$ belongs to $C$. Hence $\left(x_{n}-R x_{n}, J\left(x_{n}-u\right)\right) \geq 0$ and

$$
\left(x_{n}-x, J\left(x_{n}-u\right)\right) \leq 0
$$

for all $n \in \mathbb{N}$. On the other hand,

$$
\begin{aligned}
\left|x_{n}-u\right|^{2}=\mid x_{n}-(1-t) u & -t y+\left.t(y-u)\right|^{2} \\
& \geq\left|x_{n}-(1-t) u-t y\right|^{2}+2 t\left(y-u, J\left(x_{n}-(1-t) u-t y\right)\right)
\end{aligned}
$$

for all $0<t<1$ and each $y \in X$. Given $\varepsilon>0$, the weak-star uniform continuity of the normalized duality mapping $J$ on bounded subsets of Banach spaces with uniformly Gâteaux differentiable norms [R5, Lemma 2.2] implies that

$$
\left|\left(y-u, J\left(x_{n}-u\right)-J\left(x_{n}-(1-t) u-t y\right)\right)\right|<\varepsilon
$$

for all small enough $t$. Hence

$$
\begin{aligned}
& \left(y-u, J\left(x_{n}-u\right)\right)<\varepsilon+\left(y-u, J\left(x_{n}-(1-t) u-t y\right)\right) \\
& \quad \leq \varepsilon+\left(\left|x_{n}-u\right|^{2}-\left|x_{n}-(1-t) u-t y\right|^{2}\right) /(2 t) .
\end{aligned}
$$

Since $f$ attains its minimum at $u$, it follows that

$$
\operatorname{LIM}\left(\left\{\left(y-u, J\left(x_{n}-u\right)\right)\right\}\right) \leq 0
$$

for each $y \in X$. Choosing $y=x$ and recalling that $\left(x_{n}-x, J\left(x_{n}-u\right)\right) \leq 0$ for all $n \in \mathbb{N}$, we conclude that $\operatorname{LIM}\left(\left\{\left|x_{n}-u\right|^{2}\right\}\right) \leq 0$. Therefore there exists a subsequence of $\left\{x_{n}\right\}$ which converges strongly to $u$. Denoting $u$ by $Q x$, we obtain $(x-Q x, J(y-Q x)) \leq 0$ for all $x \in X$ and $y \in C$. Thus $Q: X \rightarrow C$ is the unique SNR of $X$ onto $C$.

Let $C$ be a nonempty, closed and convex subset of a two-dimensional Banach space $(X,|\cdot|)$. Suppose we already know (cf. [K, Theorem 2]) that $C$ is an NR of $X$ whenever the norm $|\cdot|$ is smooth. Then Proposition 5.2 shows that $C$ is, in fact, an SNR of $X$ even when $|\cdot|$ is not smooth (cf. [B2, p. 353] and Theorem 1.1). To see this, let $\left\{\varepsilon_{n}: n=1,2, \ldots\right\}$ be a positive sequence which converges to zero. For each $n \in \mathbb{N}$, let $|\cdot|_{n}$ be a smooth norm on $X$ which satisfies $|x| \leq|x|_{n} \leq\left(1+\varepsilon_{n}\right)|x|$ for all $x \in X$. By Proposition 5.2, for each $n \in \mathbb{N}$, there is an $\operatorname{SNR} Q_{n}:\left(X,|\cdot|_{n}\right) \rightarrow C$. Since $\left|Q_{n} x-Q_{n} y\right| \leq\left|Q_{n} x-Q_{n} y\right|_{n} \leq$ $|x-y|_{n} \leq\left(1+\varepsilon_{n}\right)|x-y|$ for all $x$ and $y$ in $X$, and for a fixed $y_{0} \in C,\left|Q_{n} x-y_{0}\right|=$ 
$\left|Q_{n} x-Q_{n} y_{0}\right| \leq\left|Q_{n} x-Q_{n} y_{0}\right|_{n} \leq\left|x-y_{0}\right|_{n} \leq\left(1+\varepsilon_{n}\right)\left|x-y_{0}\right|$, we can invoke the ArzelàAscoli theorem and find a subsequence of $\left\{Q_{n}\right\}$ which converges uniformly on bounded subsets of $X$ to a mapping $Q:(X,|\cdot|) \rightarrow C$. It is not difficult to check that $Q$ is indeed an $\mathrm{SNR}$ of $(X,|\cdot|)$ onto $C$, as asserted.

Now let $(X, \rho, M)$ be a hyperbolic metric space in the sense of [RS]. Given any two distinct points $x$ and $y$ in $X$, there is a unique metric line in $M$ which passes through $x$ and $y$. This metric line determines a unique metric segment joining $x$ and $y$. We denote this segment by $[x, y]$. For each $0 \leq t \leq 1$, there is a unique point $z \in[x, y]$ such that $\rho(x, z)=t \rho(x, y)$ and $\rho(z, y)=(1-t) \rho(x, z)$. This point will be denoted by $(1-t) x \oplus t y$. Also, for each $r \geq 0$, we let $(1+r) x \ominus r y$ stand for the unique point $w$ on this metric line satisfying $\rho(w, x)=r \rho(x, y)$ and $\rho(w, y)=(1+r) \rho(x, y)$.

Lemma 5.3. Let $C$ be a Chebyshev subset of a hyperbolic metric space $(X, \rho, M)$ and let $P: X \rightarrow C$ be the nearest point projection of $X$ onto $C$. Then $P(t x \oplus(1-t) P x)=P x$ for all $x \in X$ and $0 \leq t \leq 1$.

Proof. Denote $t x \oplus(1-t) P x$ by $z$. Since $\rho(x, P z) \leq \rho(x, z)+\rho(z, P z) \leq \rho(x, z)+\rho(z, P x)=$ $(1-t) \rho(x, P x)+t \rho(x, P x)=\rho(x, P x)$, we see that $P z=P x$, as claimed.

If $C$ is a retract of a hyperbolic metric space $X$ and $R: X \rightarrow C$ is a retraction of $X$ onto $C$, then we say that $R$ is sunny if $R((1-t) R x \oplus t x)=R((1+r) x \ominus r R x)=R x$ for all $x \in X, 0 \leq t \leq 1$, and $r \geq 0$.

Proposition 5.4. If $C$ is a Chebyshev subset of a hyperbolic metric space $(X, \rho, M)$ and the nearest point projection $P: X \rightarrow C$ is nonexpansive, then it is also sunny.

Proof. Fix a point $x \in X$. For $0 \leq t \leq 1$ and $r \geq 0$, denote $(1-t) P x \oplus t x$ and $(1+r) x \ominus r P x$ by $z$ and $w$, respectively. Let $s=1 /(1+r)$. Then $x=(1-s) P x \oplus s w$. Consider $y=(1-s) P w \oplus s w$. Since $P$ is the nearest point projection of $X$ onto $C$, we know by Lemma 5.3 that $P z=P x$ and $P y=P w$. Since $P$ is nonexpansive, we also have $\rho(P w, P x)=\rho(P y, P x) \leq \rho(y, x)=\rho((1-s) P w \oplus s w,(1-s) P x \oplus s w) \leq(1-s) \rho(P w, P x)$. The result now follows because $s>0$.

Proposition 5.4 applies, in particular, to Hilbert space and to the Hilbert ball. It shows that all the $(\rho)$-closed and $(\rho)$-convex subsets of these spaces are SNR's.

It is known [B1, Example 1] that Theorem 2.6 is no longer true if $T$ is not a selfmapping of $C$. It is, however, valid for weakly inward mappings. We also note the following improvement of Theorems 1 and 2 in $[\mathrm{X}]$. It provides an affirmative answer to a question raised on p. 155 there.

Let $C$ be a nonempty, closed and convex subset of a Banach space $X$. For $x \in C$, let

$$
I_{C}(x):=\{z \in X: z=x+a(y-x) \text { for some } y \in C \text { and } a \geq 0\}
$$

be the inward set of $C$ at $x$. Recall that a mapping $T: C \rightarrow X$ is said to be weakly inward if $T x$ belongs to the closure of $I_{C}(x)$ for each $x$ in $C$. Suppose now that a weakly inward $T: C \rightarrow X$ is also nonexpansive, and fix a point $x$ in $C$. Then for each $0 \leq t<1$, the strict contraction $S: C \rightarrow X$, defined by $S y:=t T y+(1-t) x$ for each $y \in C$, is also weakly inward and therefore has a unique fixed point, which we denote by $x_{t}$. 
Proposition 5.5. Let $C$ be a nonempty, closed and convex subset of a reflexive Banach space $X$ with a uniformly Gâteaux differentiable norm. Assume that every weakly compact and convex subset of $X$ has the fixed point property for nonexpansive mappings. Let the nonexpansive and weakly inward mapping $T: C \rightarrow X$ have a fixed point. Given $x \in C$ and $0 \leq t<1$, let $x_{t} \in C$ be the unique solution to the equation $w=t T w+(1-t) x$. Then the strong $\lim _{t \rightarrow 1^{-}} x_{t}=Q x$, where $Q: C \rightarrow F(T)$ is the unique SNR of $C$ onto the fixed point set of $T$.

Proof. Consider the accretive operator $A=I-T$, where $I$ denotes the identity operator on $C$. Since the strict contraction $S: C \rightarrow X$ defined above has a unique fixed point for each $x \in C$, the resolvent $J_{r}:=(I+r A)^{-1}$ is seen to be defined on all of $C$ for each $r>0$. In other words, the accretive operator $A$ satisfies the range condition $R(I+r A) \supset C$ for all positive $r$. The result now follows from Theorem 1 and Remark 1 in [R4] because $x_{t}=J_{r} x$, where $r=t /(1-t)$.

Finally, we remark in passing that if, in the setting of Proposition 5.5, $C$ itself happens to be an SNR of $X$ and $R: X \rightarrow C$ is the unique sunny nonexpansive retraction of $X$ onto $C$, then the iterative scheme of Corollary 2 in [R4] can be applied to $R T: C \rightarrow C$ to yield a fixed point of $T$. This is because any fixed point of $R T$ is, in fact, a fixed point of $T$ itself.

Acknowledgements. The first author was supported by Grants No. FWF-P16674-N12 and GAČR 201/06/0018, as well as by IRP No. AV0Z10190503. The second author was partially supported by the Technion VPR Fund, Grant No. 2005493, and by the Fund for the Promotion of Research at the Technion, Grant No. 2002146. Both authors thank the Erwin Schrödinger International Institute for Mathematical Physics in Vienna for its support.

\section{References}

[AK] L. Ambrosio, B. Kirchheim, Currents in metric spaces, Acta Math. 185 (2000), 1-80.

[Be] B. Beauzamy, Projections contractantes dans les espaces de Banach, Bull. Sci. Math. (2) 102 (1978), 43-47.

[BM] B. Beauzamy, B. Maurey, Points minimaux et ensembles optimaux dans les espaces de Banach, J. Funct. Anal. 24 (1977), 107-139.

[BP] C. Bessaga, A. Pełczyński, Selected Topics in Infinite-Dimensional Topology, Monografie Matematyczne 58, PWN, Warszawa 1975.

[Bo] F. Bohnenblust, Subspaces of $\ell_{p, n}$ spaces, Amer. J. Math. 63 (1941), 64-72.

[B1] R. E. Bruck, Properties of fixed-point sets of nonexpansive mappings in Banach spaces, Trans. Amer. Math. Soc. 179 (1973), 251-262.

[B2] R. E. Bruck, Nonexpansive projections on subsets of Banach spaces, Pacific J. Math. 47 (1973), 341-355.

[B3] R. E. Bruck, A characterization of Hilbert space, Proc. Amer. Math. Soc. 43 (1974), $173-175$.

[DE] W. J. Davis, P. Enflo, Contractive projections on $\ell_{p}$ spaces, in: Analysis in Urbana, vol. I (Urbana 1986-1987), London Math. Soc. Lecture Note Ser. 137, Cambridge Univ. Press, Cambridge 1989, 151-161. 
[D] J. Dugundji, An extension of Tietze's theorem, Pacific J. Math. 1 (1951), 353-367.

[E1] P. Enflo, Contractive projections onto subsets of $L^{1}(0,1)$, in: Analysis in Urbana, vol. I (Urbana 1986-1987), London Math. Soc. Lecture Note Ser. 137, Cambridge Univ. Press, Cambridge 1989, 162-184.

[E2] P. Enflo, Contractive projections onto subsets of $L^{p}$-spaces, in: Function Spaces (Edwardsville 1990), Lecture Notes in Pure and Appl. Math. 136, Dekker, New York 1992, $79-95$.

[FK] D. G. deFigueiredo, L. A. Karlovitz, On the extension of contractions on normed spaces, in: 1970 Nonlinear Functional Analysis, Proc. Sympos. Pure Math. 18, Part 1, Amer. Math. Soc., Providence 1970, 95-104.

[GR] K. Goebel, S. Reich, Uniform Convexity, Hyperbolic Geometry, and Nonexpansive Mappings, Monogr. Textbooks Pure Appl. Math., Marcel Dekker, New York 1984.

[G] P. M. Gruber, Fixpunktmengen von Kontraktionen in endlichdimensionalen normierten Räumen, Geometriae Dedicata 4 (1975), 179-198.

$[\mathrm{H}] \quad$ L. Hetzelt, Über die beste Coapproximation in $\mathbb{R}^{n}$, dissertation, University of ErlangenNürnberg, 1981.

[KL] A. Kamińska, G. Lewicki, Contractive and optimal sets in modular spaces, Math. Nachr. 268 (2004), 74-95.

[K] L. A. Karlovitz, The construction and application of contractive retractions in twodimensional normed linear spaces, Indiana Univ. Math. J. 22 (1972), 473-481.

[Ko] E. Kopecká, Small set in a large box, Math. Notes, to appear.

[MR] E. Matoušková, S. Reich, The Hundal example revisited, J. Nonlinear Convex Anal. 4 (2003), 411-427.

[P] R. R. Phelps, Convex sets and nearest points, Proc. Amer. Math. Soc. 8 (1957), 790-797.

[R1] S. Reich, Asymptotic behavior of contractions in Banach spaces, J. Math. Anal. Appl. 44 (1973), 57-70.

[R2] S. Reich, Extension problems for accretive sets in Banach spaces, J. Funct. Anal. 26 (1977), 378-395.

[R3] S. Reich, Product formulas, nonlinear semigroups, and accretive operators, J. Funct. Anal. 36 (1980), 147-168.

[R4] S. Reich, Strong convergence theorems for resolvents of accretive operators in Banach spaces, J. Math. Anal. Appl. 75 (1980), 287-292.

[R5] S. Reich, On the asymptotic behavior of nonlinear semigroups and the range of accretive operators, J. Math. Anal. Appl. 79 (1981), 113-126.

[R6] S. Reich, A limit theorem for projections, Linear Multilinear Algebra 13 (1983), 281-290.

[R7] S. Reich, Convergence, resolvent consistency, and the fixed point property for nonexpansive mappings, in: Fixed Points and Nonexpansive Mappings (Cincinnati 1982), Contemp. Math. 18, Amer. Math. Soc., Providence 1983, 167-174.

[RS] S. Reich, I. Shafrir, Nonexpansive iterations in hyperbolic spaces, Nonlinear Anal. 15 (1990), 537-558.

[V] L. Veselý, A characterization of reflexivity in the terms of the existence of generalized centers, Extracta Math. 8 (1993), 125-131.

[We] S. Wenger, Isoperimetric inequalities of Euclidean type in metric spaces, Geom. Funct. Anal. 15 (2005), 534-554.

[W] U. Westphal, Contractive projections on $\ell^{1}$, Constr. Approx. 8 (1992), 223-231.

[X] H.-K. Xu, Approximating curves of nonexpansive nonself-mappings in Banach spaces, C. R. Acad. Sci. Paris Sér. I Math. 325 (1997), 151-156. 